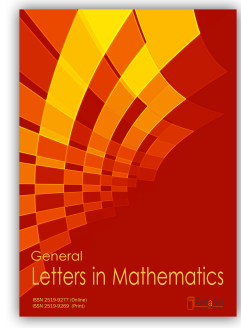

General Letters in Mathematics Vol. 2, No. 2, April 2017, pp.94-98

e-ISSN 2519-9277, p-ISSN 2519-9269

Available online at http:// www.refaad.com

\title{
Source to Coast Pollutants Distribution in Tangier Area: Arc GIS Interpolation and Finite Element Methods
}

\author{
Saiida Lazaar*1 and Siham Abourida ${ }^{2}$ \\ 12 Mathematics and Computing Department \\ Mathematics, Computer Science and Applications Research Team \\ ENSA of Tangier, AbdelMalek Essaadi University \\ ${ }^{1 *}$ s. lazaar2013@gmail. com ${ }^{2}$ siham. abourida@hotmail.fr
}

\begin{abstract}
This work attempts a contribution for studying marine pollution from a numerical point of view and aims to offer some solutions for the preservation of marine environment. For more reliability, Tangier bay (North of Morocco) was chosen as a study area. We underline that the main topic of this paper deals with numerical simulation and interpolation of hydrocarbons and heavy metals concentrations; both of these harmful pollutants are spilled into Tangier bay. We define a mathematical model built on a system of Navier-Stokes and convection-dispersion equations in order to estimate contaminant concentration by a finite element method using FreeFem ++ software. Otherwise, we perform a spatial interpolation for heavy metals concentrations in order to take into account actual data and to validate numerical tests. Finally, the paper exhibits results from both finite element and Arc GIS interpolation methods, and it is a continuation of some of our published works.
\end{abstract}

Keywords: Metadata; Arc GIS; Convection; Dispersion; Finite Element; FreeFem++; Heavy Metals; NavierStokes.

\section{Introduction}

Tangier bay is an environment strongly influenced by contaminants related to harbor, urban and industrial activities. Therefore, a good management policy of natural resources is mandatory.

In this paper, we approach the problematic of pollution from a numerical point of view. Various numerical modeling studies of pollution for different bays around the world have been carried out. The paper [2] reported a detailed survey of worldwide bays marine pollution, and a state of the art of related numerical methods. Here, we present advanced results of already published works $([1],[4])$. The new results deal with numerical simulation and spatial interpolation of hydrocarbon and heavy metal concentrations. The mathematical model is built on transport-dispersion equations and hydrodynamic process. The latter is established from Navier-Stokes equations giving velocity and prevailing swells current trends. The global model is obtained from convection-dispersion equations which simulate transport and dispersion of pollutants discharged into Tangier Bay. We remind that the convection term is obtained by solving characteristics equation while the temporal term is approximated with an implicit Euler scheme. The obtained variational problem is then solved by a finite element method with FreeFem++ software.

To strengthen this work, we analyze the actual data collected from field and we realize an Arc GIS interpolation for pollutants concentration along rivers supplied into the bay. This interpolation is helpful to highlight source to coast pollutants distribution.

\footnotetext{
${ }^{*}$ Corresponding author.Saiida LAZAAR ${ }^{1}$ s.lazaar2013@gmail.com @ (i)
} 2010 Mathematics Subject Classification: 35A15, 65M22, 65M60. 
The paper is organized as follows. Section 2 describes mathematical modeling and numerical discretization of the problem. Section 3 presents numerical and spatial interpolation results. Section 4 closes the paper with some concluding remarks.

\section{Mathematical modeling and numerical discretization}

The physical problem is expressed by coupling two mathematical models: The first one describes the hydrodynamic process that calculates the transport velocity. The second model expresses transport and dispersion phenomenon to estimate pollutant concentration.

In the case of an incompressible flow on a study area $\Omega \subset \mathbb{R}^{3}$, the hydrodynamic process is expressed as follows:

$$
\left\{\begin{array}{l}
\left.\frac{\partial U}{\partial t}+U \cdot \nabla U+\frac{1}{\rho} \nabla P=\nu \Delta U-\frac{\tau}{h} \quad \text { in } \quad \Omega \times\right] 0, T[ \\
\left.\frac{\partial h}{\partial t}+\nabla \cdot(h U)=0 \quad \text { in } \quad \Omega \times\right] 0, T[,
\end{array}\right.
$$

where the function $U$ is the velocity, $\nu$ is the eddy viscosity of water, $\rho$ is density, $P$ denotes the pressure and it corresponds to $P=\rho g h$ with $g$ the gravity, $h$ the sea water depth and $\tau$ corresponds to the shear stress.

On the other hand, the dispersion-convection process is given by:

$$
\left\{\begin{array}{l}
\left.\frac{\partial c}{\partial t}+\operatorname{div}(U c-D \overrightarrow{g r a d} c)=f \quad \text { in } \quad \Omega \times\right] 0, T[ \\
c(., 0)=c^{0} \quad \text { in } \quad \Omega
\end{array}\right.
$$

where $c$ is the pollutant concentration in sea water, $f$ is a source term, $T$ is the observation final time and $D$ is the tensor of dispersion.

Neglecting shear stress effect on the bottom and the water height, the mathematical model adopted in this study is the following:

$$
\left\{\begin{array}{l}
\left.\frac{\partial U}{\partial t}+U . \nabla U+\frac{1}{\rho} \nabla P=\nu \triangle U \quad \text { in } \quad \Omega \times\right] 0, T[ \\
\nabla . U=0 \quad \text { in } \quad \Omega, \\
\left.\frac{\partial c}{\partial t}-\nabla \cdot(D \nabla c-U c)=f \quad \text { in } \quad \Omega \times\right] 0, T[.
\end{array}\right.
$$

For the numerical resolution of system (3), we apply an implicit Euler scheme and a variational formulation with a finite element discretization.

For the time discretization of the hydrodynamic model, we introduce the particular derivative to approach the convection term $\frac{\partial U}{\partial t}+U . \nabla U$ by the following characteristics equation:

$$
\frac{\partial U}{\partial t}+U \cdot \nabla U \simeq \frac{U^{n+1}-U^{n} \circ X^{n}}{\Delta t}
$$

where $\Delta t$ is the time step and $X^{n}(x)$ is the function which gives the position of the particle $x$ at time $n \Delta t$. Therefore, we obtain the following discretization scheme:

$$
\left\{\begin{array}{l}
U^{n+1}-\nu \Delta t \Delta U^{n+1}+\Delta t \nabla P^{n+1}=U^{n} \circ X^{n} \\
\nabla \cdot U^{n+1}=0
\end{array}\right.
$$

By introducing $\omega$ and $q$ as test functions in $H^{1}(\Omega)^{2}$ and $L^{2}(\Omega)$, we obtain:

$$
\left\{\begin{array}{l}
\frac{1}{\Delta t} \int_{\Omega} U^{n+1} \cdot \omega-\nu \int_{\Omega} \Delta U^{n+1} \cdot \omega+\int_{\Omega} \nabla P^{n+1} \cdot \omega=\frac{1}{\Delta t} \int_{\Omega}\left(U^{n} \circ X^{n}\right) \cdot \omega \\
\int_{\Omega} q\left(\nabla \cdot U^{n+1}\right)=0 .
\end{array}\right.
$$


Thereafter, we obtain the following variational problem ready to be implemented by FreeFem ++ code ([3]): For a given function $U^{n} \in V_{h}$, find the functions $\left(U^{n+1}, P^{n+1}\right) \in V_{h} \times M_{h}$ such that:

$$
\left\{\begin{array}{l}
\forall \omega \in V_{h}, \quad a\left(U^{n+1}, \omega\right)+b\left(\omega, P^{n+1}\right)=l(\omega), \\
\forall q \in M_{h}, \quad b\left(U^{n+1}, q\right)=0
\end{array}\right.
$$

where

$$
\left\{\begin{array}{l}
a\left(U^{n+1}, \omega\right)=\frac{1}{\Delta t} \int_{\Omega} U^{n+1} \omega+\nu \int_{\Omega} \nabla U^{n+1} \nabla \omega, \\
b\left(\omega, P^{n+1}\right)=-\int_{\Omega} P^{n+1} \nabla \omega, \\
l(\omega)=\frac{1}{\Delta t} \int_{\Omega}\left(U^{n} \circ X^{n}\right) \omega .
\end{array}\right.
$$

We specify that $V_{h}$ and $M_{h}$ are finite element spaces constructed on regular triangulation.

In order to find the main unknown of the problem which is the concentration $c$, we choose to examine equation (2) under this formulation:

$$
\left.\frac{\partial c}{\partial t}+U \nabla c-D \Delta c=0, \quad \Omega \times\right] 0, T[
$$

Following the foregoing reasoning, we obtain the following variational problem where $\varphi$ is a test function:

$$
\forall \varphi \in H_{0}^{1}(\Omega), \frac{1}{\Delta t} \int_{\Omega} c^{n+1} \cdot \varphi-D \int_{\Omega} \Delta c^{n+1} \cdot \varphi=\frac{1}{\Delta t} \int_{\Omega} c^{n} \circ X^{n} \cdot \varphi,
$$

which is rewritten as follows:

For a given function $c^{n} \in Q_{h}$, find the function $c^{n+1} \in Q_{h}$, satisfying:

$$
\forall \varphi \in Q_{h}, \quad a\left(c^{n+1}, \varphi\right)=l(\varphi)
$$

where $Q_{h}$ denotes a finite element space for the concentration generated by a regular triangulation of $\Omega$, and

$$
\left\{\begin{array}{l}
a\left(c^{n+1}, \varphi\right)=\frac{1}{\Delta t} \int_{\Omega} c^{n+1} \varphi+D \int_{\Omega} \nabla c^{n+1} \nabla \varphi \\
l(\varphi)=\frac{1}{\Delta t} \int_{\Omega}\left(c^{n} \circ X^{n}\right) \varphi .
\end{array}\right.
$$

\section{Results and discussion}

In this paper, we present two kind of results; those obtained with a numerical resolution of the equations by a finite element method performed under FreeFem ++ software, which also elaborates the bay mesh taking into account multiple pollution sources supplied from various rivers. Other ones are performed by Arc GIS interpolation.

Figure 3 shows mesh effect on numerical resolution quality. We remark obviously that numerical resolution results in the case of fine mesh are more precise than those associated to coarse mesh.

To make a contribution for solving marine pollution in Northern Morocco, it may be important to determine sources and fate of heavy metals discharged into Tangier bay. Crucial information could be taken from Figures 3 and 3.

Figure 3 represents copper concentrations interpolation along the rivers discharged into this bay. We plot on the same figure finite element simulation results, reinforcing those given by Arc GIS.

Figure 3 presents at the left side hydrocarbon concentrations calculated by finite element method. On the right side, we present zinc concentrations interpolation along the rivers spilled in the bay and concentrations calculated by finite element method inside the bay.

We notice that highest concentrations are located between the center and the downstream of Mghogha and Souani rivers, which receives wastes deriving from Mghogha industrial area and from some factories located around Moulay Ismail avenue. However, concentrations registered along Mlaleh and Chatt rivers are small, and show an homogeneous distribution due to the absence of a polluting source in these streams. 
Numerical simulation results related to copper transport-dispersion show that highest concentrations are registered at Mghogha and Souani rivers mouth, followed by those registered at of Mlaleh and Chatt rivers mouth; these concentrations are gradually decreasing from the coast under the effect of transport-dispersion process.
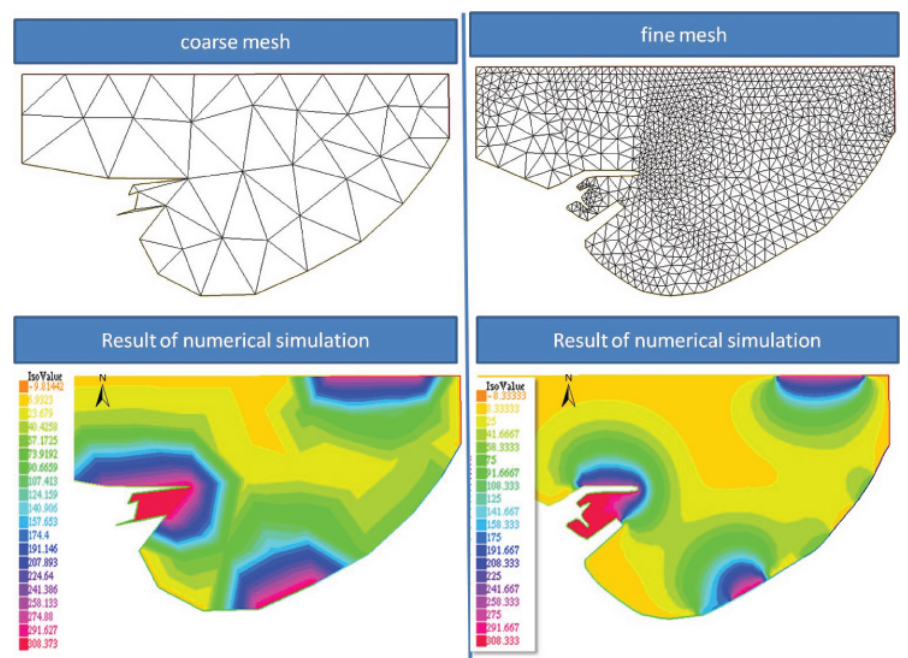

Figure 1: Mesh effect on the numerical resolution quality

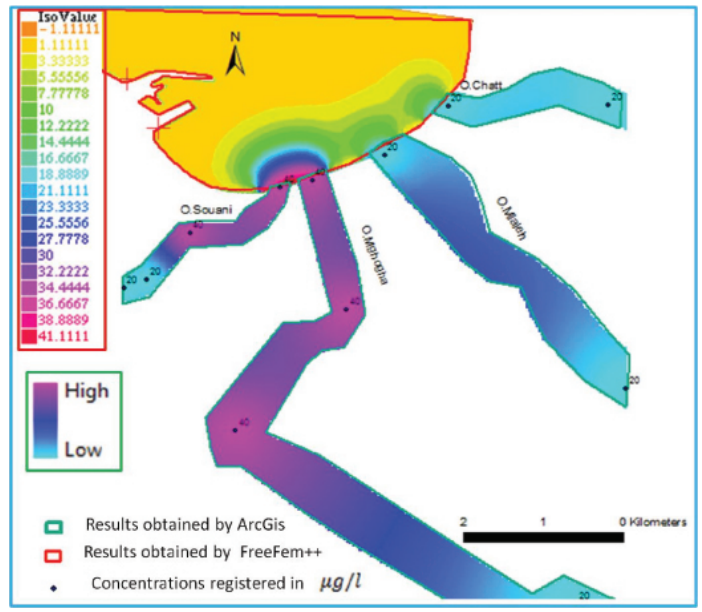

Figure 2: Copper concentrations with both numerical simulation and interpolation 

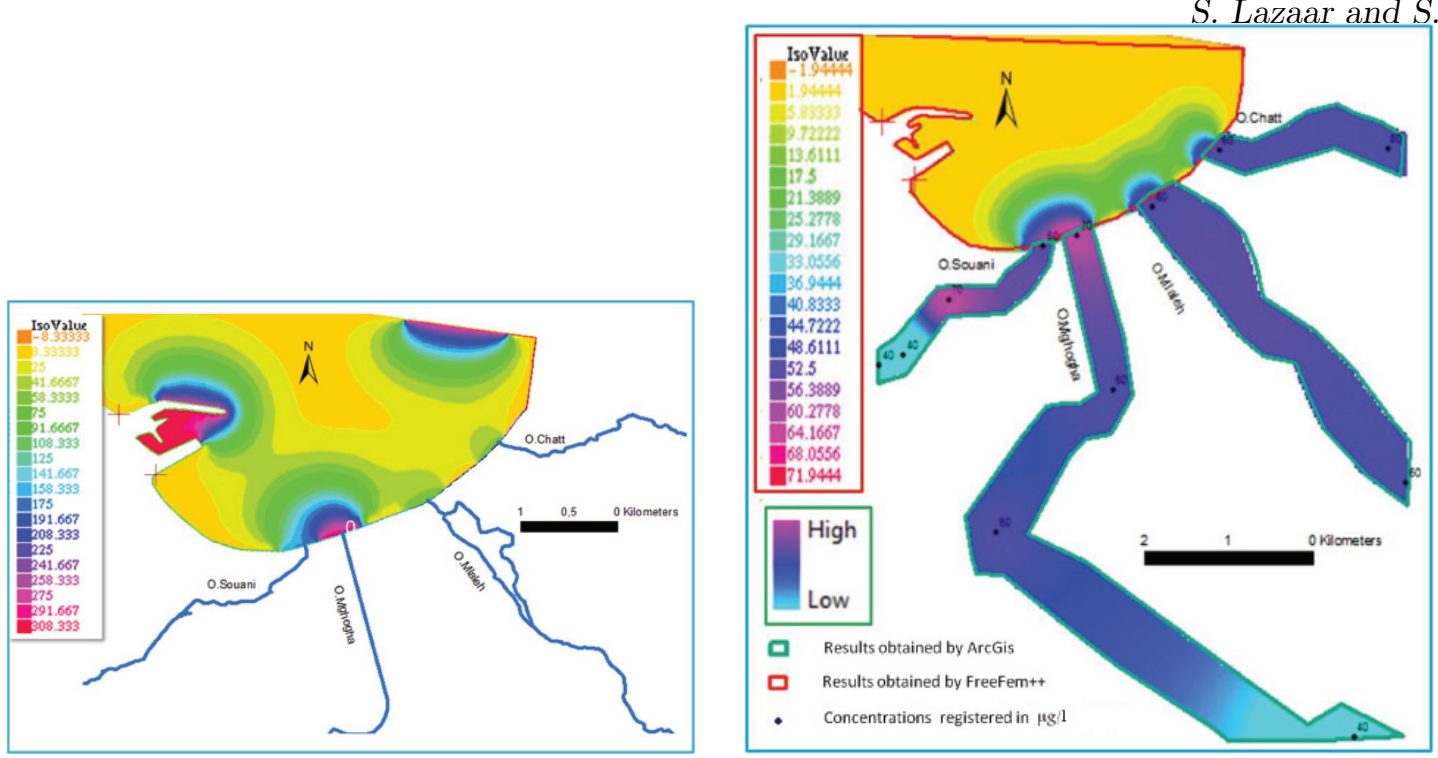

Figure 3: (left) Hydrocarbon concentrations calculated by finite element method. (right) Zinc concentrations with both numerical simulation and spatial interpolation

\section{Concluding remarks}

This work aimed at tracking of hydrocarbons and heavy metals spilled into the bay. We defined and solved a mathematical model built on Navier-Stokes and convection-dispersion equations. To validate the tests, we presented various results obtained from a numerical simulation performed with a finite element discretization, and other ones given by Arc GIS interpolation.

The numerical simulation results demonstrated that highest pollutant concentrations are set at the rivers mouth, mostly at Mghogha river. According to the spatial interpolation results, we have demonstrated that highest concentrations are located between the center and the mouth of Mghogha river which is feeded by the industrial zone. We highlight a strong compatibility between numerical simulation and spatial interpolation results mainly at the rivers mouth.

We can conclude that Tangier bay is vulnerable to numerous contaminants and this vulnerability requires a strong management policy of natural ressources.

\section{References}

[1] S. Abourida, Finite element mathematical modeling and numerical simulation of contaminants transportdispersion in Tangier Bay, PhD thesis, University AbdelMalek Essaadi, Morocco, (2016).

[2] S. Abourida, S. Lazaar and M.N. Zaghloul, "Numerical modeling of marine pollution: Application to hydrocarbons distribution in Tangier Bay", Journal of Mathematical Analysis, Vol.7, (2016), pp. 4-11.

[3] F. Hecht, O. Pironneau, J. Morice, A. Le Hyaric and K. Ohtsuka, FeeFem++, Third Edition Version, 3.10-2, (2010).

[4] S. Lazaar, S. Abourida, E. Chacón Vera and H. Er-Raioui, "Numerical simulation of hydrocarbons dispersion in Tangier Bay", Advances in Dynamical Systems and Applications, Vol. 8, (2013), pp. 75-84. 